\section{Humoral immune activation in amyotrophic lateral sclerosis patients}

\author{
Michael Rentzos, ${ }^{1}$ \\ Maria Eleftheria Evangelopoulos, \\ Eleni Sereti,2 Vassiliki Zouvelou, 1 \\ Styliani Marmara,2 Theodoros Alexakis, ${ }^{1}$ \\ loannis Evdokimidis ${ }^{1}$ \\ 1Department of Neurology, Aeginition \\ Hospital, Athens National University, \\ School of Medicine; 2Immunology \\ Department, Alexandra Hospital, Greece
}

\section{Abstract}

There is evidence that immunological factors may involved in the pathogenetic mechanisms of amyotrophic lateral sclerosis (ALS). Few studies to date have explored the status of the humoral immune response in patients with ALS. We examined the presence of humoral immune activation in ALS patients, serum immunoglobulins (IgG, IgA and IgM) levels were measured in 36 patients with ALS and 35 normal controls. Serum IgG, IgM and IgA levels were not significantly different in our ALS patients compared with the control group $(\mathrm{P}=\mathrm{ns})$. No correlations of serum $\operatorname{IgG}$, IgM and IgA concentrations with duration, severity of the disease or the clinical form of onset (bulbar or spinal) were found in our ALS patients. Our results do not suggest a humoral immune activation in ALS patients. This does not exclude that immunological mechanisms may be involved in ALS pathogenesis.

\section{Introduction}

Amyotrophic lateral sclerosis (ALS) is a devastating neurological disorder which affects upper and lower motoneurones leading to muscle weakness and wasting, respiratory depression and death. A variety of immunological abnormalities have been reported in some patients with ALS. It has been postulated that a disturbance of immunoregulation may play a role in the degeneration of motor neurons in ALS. The results of various studies analyzing serum antibody levels are contradictory. Significant increases of serum IgG, but not IgM or IgA, have been found in ALS patients. ${ }^{1}$ Besides, other reports demonstrated normal serum IgG, IgM, and IgA levels or normal IgG and high IgM levels in ALS patients at early disease stage..$^{2,3}$ In order to examine whether peripheral humoral immune activation is observed in ALS patients, we measured the serum immunoglobulins IgG, IgA and IgM concentrations in patients with ALS and normal controls. We also evaluated whether the peripheral humoral immune activation state in ALS patient was correlated with the duration, the disability level and the clinical subtype of the disease.

\section{Materials and Methods}

\section{Subjects}

After obtaining an Institutional Board Approval, serum IgG, IgM and IgA levels in 36 patients with definite or probable ALS were measured (19 men and 17 women, mean age $59.2 \pm 10.9$ years) and 35 age-matched healthy subjects (20 men and 15 women, mean age $56 \pm 11.6$ years) used as control group.

Patients with evidence of systemic inflammation on clinical examination or serum biochemical tests (increased number of white blood cells, high levels of C-reactive protein or erythrocyte sedimentation rate) were excluded. Patients with use of non-steroidal antiinflammatory drugs, acetylsalicylic acid, steroids or statins during the last two months before enrollment were also excluded from the study. All patients were submitted to appropriate blood tests to exclude ALS with Laboratory Abnormalities of Uncertain Significance (ALSLAUS) Syndromes such as monoclonal gammopathy, auto-antibodies (high-titer GM1 ganglioside antibody), non-malignant endocrine abnormalities and infection (HIV-1, varicellazoster, syphilis, borrelliosis).

In the ALS group, all patients met the revised EI Escorial criteria for possible, ${ }^{4}$ probable and definite ALS. Ten out of our patients with bulbar signs had bulbar onset. Duration of symptoms at the time of examination was $23.17 \pm 32.7$ months. The disability level associated with the development and progression of ALS was determined using the revised ALS functional-rating scale (ALSFRS-R). 5 The ALSFRS-R score was $40.3 \pm 6.5$.

Informed consent had been obtained by all patients for studying serum. Serum samples were stored at $-70^{\circ} \mathrm{C}$ until assayed.

\section{Methods}

Immunochemical determination of IgG, IgA and IgM serum levels was made with the use of Behring Nephelometer II (DADE BBEHRING, MARBURG GMBH). Serum samples were put in cuvettes along with the corresponding antiserum and the antigen-antibody complexes were formed. A light beam generated by means of an LED was scattered by the antigen-antibody complexes which were present and it was then converted, by a detector, into an electrical
Correspondence: Maria Eleftheria Evangelopoulos, Department of Neurology, Aeginition Hospital-Athens Medical School, 72-74 Vas. Sophias Av, Athens 11528, Greece.

Tel. +30.2107289200

E-mail: evangelopoulos@yahoo.com

Key words: IgM, IgG, IgA, amyotrophic lateral sclerosis, humoral immune activation.

Received for publication: 25 June 2012. Accepted for publication: 18 October 2012.

This work is licensed under a Creative Commons Attribution NonCommercial 3.0 License (CC BYNC 3.0).

(C) Copyright M. Rentzos et al., 2013

Licensee PAGEPress, Italy

Neurology International 2013; 5:e3

doi:10.4081/ni.2013.e3

signal proportional to the protein concentration in the measured sample. The final protein concentration was calculated with the use of a reference curve that had been made after several measurements of a standard with a known antigen concentration at different dilutions. The use of large latex particles led to the formation of large antigen-antibody complexes $(>1000 \mathrm{~nm}$ ) which, with the use of a smaller electromagnetic wave $(840 \mathrm{~nm})$, resulted in Mie scattering (the light was mainly scattered in the forward direction) and allowed improved light efficiency for the measurement. A prereaction test was incorporated into our protocol in order to exclude the possibility of samples with a very high antigen content giving false low results: if positive, measurement was repeated automatically at the next dilution.

\section{Statistical analysis}

Data of patients and controls were expressed as mean \pm SD. Serum immunoglobulin levels between ALS patients and controls were compared with Student $\mathrm{t}$ test. Correlations between variables were performed by the non-parametric Spearmann correlation coefficient test. A P-value of $<0.05$ was considered statistically significant. Statistical analysis was performed with the software package PRISM, CA, USA.

\section{Results}

We evaluated the immunoglobulin levels in ALS patients and were compared to those of healthy controls. Serum IgG, IgM and IgA levels were not significantly different in ALS patients compared to the control group ( $\mathrm{P}=\mathrm{ns}$ ) (Table 1). Also, no correlation of serum IgG, IgM and 
Table 1. Immunoglobulin levels in serum (mean \pm standard deviation) of amyotrophic lateral sclerosis patients and controls.

\begin{tabular}{lccc} 
Immunoglobulins & $\begin{array}{c}\text { Amyotrophic lateral } \\
\text { sclerosis patients }(\mathrm{n}=36)\end{array}$ & Controls $(\mathrm{n}=35)$ & $\mathrm{P}$ \\
IgG & $1068 \pm 196.4$ & $1141 \pm 293.9$ & $\mathrm{P}=0.16=\mathrm{NS}$ \\
IgA & $237.8 \pm 112.6$ & $213.8 \pm 88.81$ & $\mathrm{P}=0.292=\mathrm{NS}$ \\
\hline IgM & $88.6 \pm 50.5$ & $104.5 \pm 86.13$ & $\mathrm{P}=0.3=\mathrm{NS}$ \\
\hline NS, not significant. & &
\end{tabular}

Table 2. Correlations of immunoglobulin levels of amyotrophic lateral sclerosis patients with clinical parameters.

\begin{tabular}{lcc} 
Immunoglobulins & ALS FRS rho/P & Duration rho/P \\
IgG & $0.02 / 0.90$ & $0.03 / 0.85$ \\
IgA & $-0.10 / 0.60$ & $-0.01 / 0.92$ \\
\hline IgM & $-0.23 / 0.22$ & $0.02 / 0.90$ \\
\hline
\end{tabular}

ALS, amyotrophic lateral sclerosis; FRS, functional-rating scale.

Table 3. Immunoglobulin levels (mean \pm standard deviation) in serum of amyotrophic lateral sclerosis patients with and without bulbar onset.

\begin{tabular}{lccc} 
Immunoglobulins & $\begin{array}{c}\text { AIS patients without } \\
\text { bulbar onset }\end{array}$ & $\begin{array}{c}\text { AIS patients with } \\
\text { bulbar onset }\end{array}$ & P \\
IgG & $995.2 \pm 320.4$ & $1111 \pm 116.3$ & $\mathrm{P}=0.3=\mathrm{NS}$ \\
IgA & $210.9 \pm 110.3$ & $223.5 \pm 78.87$ & $\mathrm{P}=0.5=\mathrm{NS}$ \\
\hline IgM & $87.66 \pm 58.76$ & $84.56 \pm 39.40$ & $\mathrm{P}=0.7=\mathrm{NS}$ \\
\hline ALS, amyotrophic lateral sclerosis; NS, not significant. & &
\end{tabular}

IgA levels with the duration and the severity of the disease was found in ALS patients (Table 2). The levels of IgG, IgA and IgM in serum didn't differ significantly in ALS patients with or without bulbar onset (Table 3 ).

\section{Discussion and Conclusions}

In our study, the levels of serum IgG, IgM and IgA immunoglobulins were not significantly different compared to normal controls. The relative early stage of disease of our patients may explain our findings as in the study of Zhang et al. ${ }^{3}$ The absence of systemic humoral immune response found in our study, does not exclude the involvement of the immune system in ALS pathogenesis. Alterations of serum immunoglobulins have been reported in patients with motor neuron disorders and particularly in ALS,6,7 while another study showed that serum IgGs were in normal range in ALS patients and didn't differ from levels in controls. ${ }^{2}$ Appel et al. suggested that an autoimmune process may be involved in ALS since IgGs from ALS patients transferred physiological abnormalities to the neuromuscular junction in experimental animals. ${ }^{8}$ Possible targets for the antibodies would be voltage-dependent calcium channels. ${ }^{9}$ Other studies showed the relationship between serum antibodies and neuron injury related glutamate release in ALS.10

Serum immunoglobulins levels (Igs) were not correlated to the duration, the severity or the clinical form of the disease. However, some studies showed a correlation between between Igs levels and the progression of the disease. ${ }^{3,11}$ These results as well as the change of circulating immunocomplexes in some ALS patients may suggest a probable humoral immune response against undetected infections. ${ }^{11}$

The contradictory results of serum Igs concentrations in ALS patients may be due to different methods and to varying test sensitivities. Serial measurements of the status of Igs activation in patients with ALS might provide useful informations about the course of the disease and the role of humoral immune mechanisms in ALS pathogenesis.

\section{References}

1. Provinciali L, Laurenzi MA, Vesprini L, et al. Immunity assessment in the early stages of amyotrophic lateral sclerosis: a study of virus antibodies and lymphocyte subsets. Acta Neurol Scand 1988;78:449-54.

2. Bartfeld H, Dham C, Donnenfeld H, et al. Immunological profile of amyotrophic lateral sclerosis patients and their cell-mediated immune responses to viral and CNS antigens. Clin Exp Immunol 1982;48:137-46.

3. Zhang R, Gascon R, Miller R, et al. Evidence for systemic immune system alterations in sporadic amyotrophic lateral sclerosis (sALS). J Neuroimmunol 2005;159:215-24.

4. Brooks M, Miller R, Swash M, Munsat TL. El Escorial revisited: revised criteria for the diagnosis of amyotrophic lateral sclerosis. Amyotroph Lateral Scler 2000;1:293-9

5. Cedarbaum J, Stambler N, Malta E, et al. The ALSFRS-R: a revised ALS functional rating scale that incorporates assessments of respiratory function. J Neurol Sci 1999;169:13-21.

6. Younger DS, Rowland LP, Latov N, et al. Lymphoma, motor neuron diseases, and amyotrophic lateral sclerosis. Ann Neurol 1991;29:78-86.

7. Duarte F, Binet S, Lacomblez L, et al. Quantitative analysis of monoclonal immunoglobulins in serum of patients with amyotrophic lateral sclerosis. J Neurol Sci 1991;104:88-91.

8. Appel SH, Engelhardt JL, Garcia J, Stefani E. Immunoglobulins from animal models of motor neuron disease and from human amyotrophic lateral sclerosis patients passively transfer physiological abnormalities to the neuromuscular junction. Proc Nat Acad Sci USA 1991;88:647-51.

9. Pagani MR, Reisin RC, Uchitel OD. Calcium signaling pathways mediating synaptic potentiation triggered by amyotrophic lateral sclerosis IgG in motor nerve terminals. $\mathrm{J}$ of Neurosci. 2006;26: 2661-72.

10. La Bella V, Goodman JC, Appel SH. Increased CSF glutamate following injection of ALS immunoglobulins. Neurol 1997;48:1270-2.

11. Saleh I, Zesiewicz T, Xie Y, et al. Evaluation of humoral immune response in adaptive immunity in ALS patients during disease progression. J Neuroimmunol 2009;215:96-101. 